\title{
OUTPUT IMPEDANCE IMPROVEMENT USING COUPLED INDUCTORS
}

\author{
E. Sanchis Kilders, A. Ferreres, J.L. Gasent Blesa, D. Osorno, D. Gilabert, \\ E. Maset, J. Jordán, V. Esteve, J.B. Ejea \\ Dpt. Ingeniería Electrónica / ETSE, Universidad de Valencia, Avd. de la Universitat s/n, 46100 Burjassot, Spain, \\ Email: esteban.sanchis@uv.es
}

\begin{abstract}
When using a single DC/DC converter with multiple outputs and having a buck topology, which has one filter inductor per output, the designer can choose to couple these outputs together. This paper demonstrates additional benefits of coupling output inductors together. Apart from saving mass and volume, and due to an improved small signal behaviour it also reduces the output impedance of the regulated output. The paper will analyse a seven output push-pull converter used as a space power converter module and verify the theoretical results with experimental measurements.
\end{abstract}

Key words: small signal analysis; output impedance; coupled inductors; peak current control.

\section{INTRODUCTION}

Coupled inductors applied to interleaved topologies have already been studied in [1] [2] [3] [4]. Their use in multiple output converters, usually applying weighted control, is studied in [1] [5] [6] [7] and an improvement of cross regulation has been demonstrated in [5]. Coupled inductors also save volume and mass, which for some applications, like space-borne systems, is definitely an important advantage. A small signal analysis of a coupled inductor forward converter is presented in [6] and the transfer functions for a two outputs case are studied. A study including peak current mode control is presented in [7], providing information not only on the transfer function but also on output impedance and even cross impedance. Other uses of coupled inductors like in photovoltaic systems and its advantages are described in [8], where zero ripple in the source current is achieved by design. In [9], the authors propose to use coupled inductors to improve the transient response of high power output feeding load voltage / high current loads. It is a way of taking advantage of the lower output impedance of coupled inductor converters.

This paper presents a detailed study of the output impedance of seven coupled inductors used together with primary peak current control in a push-pull converter.
The model has been simulated and experimentally validated with a converter that is the Power Converter Module (PCM) of a real space application. A detailed description and more practical information of the PCM of the Polarimetric and Helioseismic Imager (PHI), instrument to fly on board of the Solar Orbiter satellite (SolO) was already published in [10].

\section{POWER SUPPLY OF THE PHI INSTRUMENT}

Although already mentioned in [10] and for the sake of completeness, a short description of the PCM designed for PHI to fly onboard SolO will be given. The PCM is based on a push-pull topology with seven outputs using a single transformer and a single output coupled inductor (see Fig. 1), mainly to save volume, mass and to improve small signal behaviour and output impedance, as this paper will demonstrate.

The PCM is a custom design system providing seven output voltages $(+3.4 \mathrm{~V},+7 \mathrm{~V},-7 \mathrm{~V},+14 \mathrm{~V},-14 \mathrm{~V}$, $+65 \mathrm{~V}$ and primary auxiliary $+14 \mathrm{~V}$ ) with galvanic isolation. Two $+28 \mathrm{~V}$ output voltages are also delivered from the PCM primary side ( $28 \mathrm{~V}$ bus voltage), but are not considered in the study, as they do not pass through the DC/DC converter. The PCM manages a maximum power of around $35 \mathrm{~W}$. The PCM not only includes the DC/DC converter, but also protections, inrush current control, dedicated input filter and a whole power distribution unit on its output to properly supply all required voltages to each user.

The engineering model (EM) of the PCM was already built and is shown in Fig. 2. The EM of the PCM was used to measure the experimental results presented at the end of the paper. Therefore, not just a simple breadboard was used to validate the theoretical results but a real space application subsystem. This has allowed to verify the effect of all additional circuitry on the small signal analysis and output impedance and how to model this effect in a SPICE simulation. 


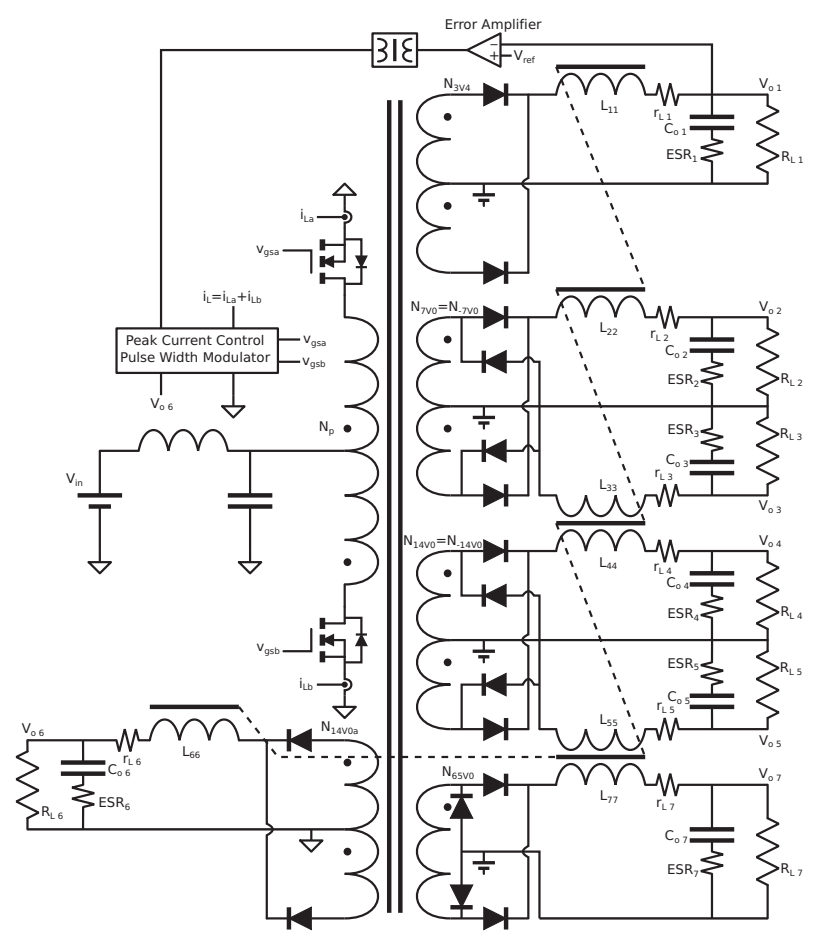

Figure 1. Simplified schematic of the PCM converter.

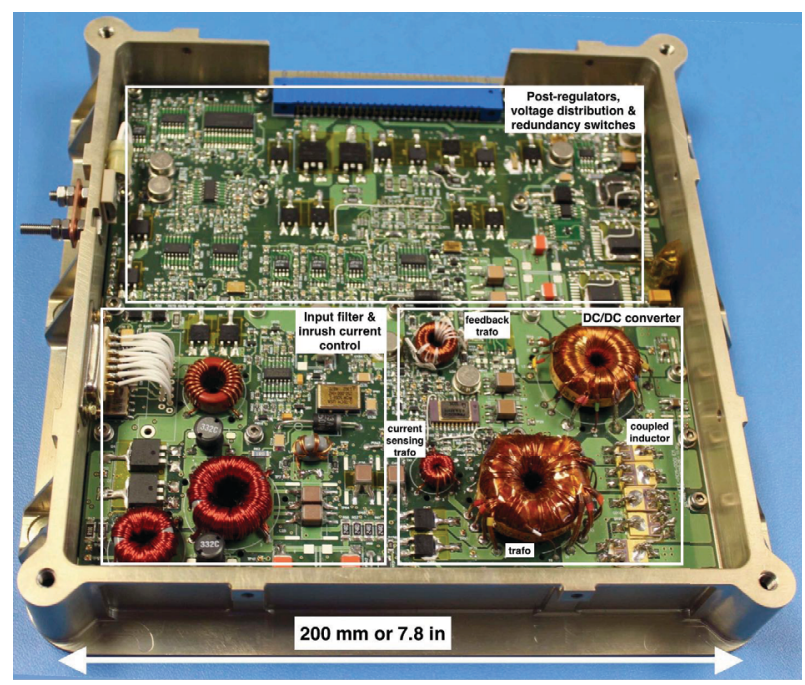

Figure 2. Engineering Model of the PCM. On the right side of the picture, the coupled inductor can be seen and below it the main transformer. The three ring cores on the left belong to the input filter and the two small ring cores in the middle are the magnetic feedback and the current sensing transformer.

\section{SMALL SIGNAL ANALYSIS}

The theoretical study has been focused on the coupled inductor, which therefore has been considered real. The model used for the coupled inductor is the inductance matrix, which mathematically suits best for the whole equations deduction and is the real and exact description of a coupled inductor (but neglecting parasitic elements, like capacitances). Measuring the inductance matrix has to be done very carefully [1] [11] and the matrix will be physically feasible if, and only if, its eigenvalues are real and positive (demonstrated in [12]).

\subsection{Procedure}

The listed steps have been followed:

1. The small signal analysis of a simplified BUCKtype converter with coupled inductors has been done.

2. The linearised model has been studied including the experimentally measured coupling inductance matrix. The resulting equations can be particularised for no coupling (changing all mutual inductances of the inductance matrix to zero).

3. A linearised model has been simulated with SPICE to validate the theoretical model (see Fig. 8) and the transfer functions have been compared graphically to the previous obtained equations for verification purposes.

4. A second and more complete SPICE circuit (based on the previous one and shown in Fig. 9) has been built incorporating many additional parasitic and real components, like input filter, duty cycle loss due to the transformer's leakage, parasitic resistance of tracks of the PCB and of the current sensing shunts used in the PCM among others.

5. The frequency response of the complete SPICE model and the real circuit measurement has been compared.

\subsection{Analysis}

The small signal analysis is based on well-known averaging techniques. Taking into account that the Push-Pull converter is a BUCK type topology and that in our case all output inductors are coupled together, only the secondary side of the $j$-th output, shown in Fig. 3, is used for the analysis.

In Fig. 3, rectifying diodes have already been linearised and the input transformer has also been converted into a DC transformer, that includes not only the turns ratio but also the effect of the duty cycle. Using now matrix algebra to analyse the circuit, we can take into account that all outputs are coupled together and therefore interact with each other. The sub-indices of the matrices are $j$ and $k$ and both vary from 1 to 7 . Of course, the dimensions of the matrices that appear in these formulae are equal to the number of coupled inductors of the converter and are equal to seven in our case. Once the inductance matrix, $\mathbf{L}_{\mathbf{o}}$, is known (including mutual inductances, $L_{j k}$ and self 


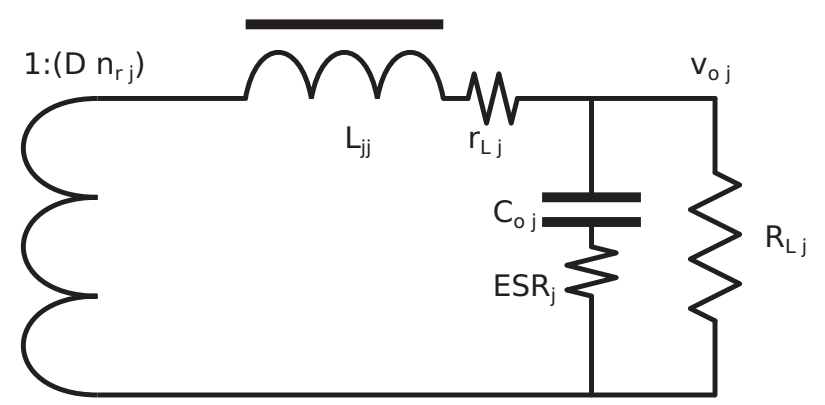

Figure 3. Small signal model of the $j$-th output of the push-pull converter. $D$ is the duty cycle, $n_{r} j$ is the turns ratio, $v_{o}$ is the output voltage, $L_{j j}$ is the self inductance, $C_{o j}$ is the output capacitor, $R_{L} j$ is the load resistance, $r_{L} j$ is the parasitic series resistance of the inductance, $E S R_{j}$ is the equivalent series resistance of the output capacitor.

inductances, $L_{j j}$ ), it has to be checked if the matrix is physically feasible, i.e. it is a semidefinite positive matrix [12].

The block diagram that describes the small signal behaviour is shown in Fig. 4.

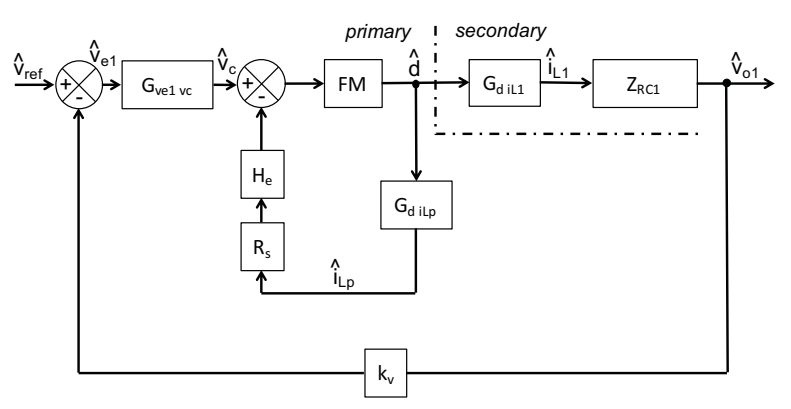

Figure 4. Small signal control block diagram of the PCM. The peak current control is closed measuring the primary current, $i_{L}$, which sums up all secondary currents with their corresponding transformer ratio.

The expressions for the different blocks shown in Fig. 4 are given hereafter.

$H_{e}$ represents the sampling effect of peak current control and its expression (Eq. 1) is demonstrated in literature. $F M$ (Eq. 2) corresponds to the modulators transfer function found in a peak current control loop, where $f_{s}$ is the switching frequency, $R_{s}$ is the current sensing resistor, $V_{i n}$ is the input voltage and $L_{p}$ is the equivalent primary inductance.

$$
\begin{gathered}
H_{e}(s)=\frac{\frac{s}{f_{s}}}{e^{\frac{s}{f_{s}}}-1} \approx 1-\frac{\frac{s}{f_{s}}}{2}+\left(\frac{\frac{s}{f_{s}}}{\pi}\right)^{2} \\
F M=\frac{f_{s}}{R_{s} \frac{V_{i n}}{L_{p}}}
\end{gathered}
$$

$G_{d i_{L}}$ is the transfer ratio related to primary current $i_{L}$ and duty cycle $d$.

$$
G_{d i_{L}}(s)=\sum_{j} n_{r j} G_{d i_{L} j}(s)
$$

where the transfer function of the $j$-th output related to the primary current is,

$$
G_{d i_{L} j}(s)=\frac{V_{i n} Y_{L R C p j}(s)}{n_{r j}}
$$

The transformer transfer ratio is given by $n_{r}$, where the general expression of the transposed vector, $\mathbf{n}_{\mathbf{r}}^{\mathbf{T}}$, is given by

$\mathbf{n}_{\mathbf{r}}^{\mathbf{T}}=\frac{1}{N_{p}}\left(N_{3 V 4} N_{7 V 0} N_{-7 V 0} N_{14 V 0} N_{-14 V 0} N_{14 V 0 a} N_{65 V 0}\right)$

The admittance $Y_{L R C p j}$ of each output seen from the primary side, corresponds to

$$
Y_{L R C p j}(s)=n_{r j} \sum_{k} n_{r k}\left[\mathbf{Z}_{\mathbf{L R C}}(s)^{-1}\right]_{j k}
$$

Where the impedance $Z_{L R C ~ j k}$ is given by,

$$
Z_{L R C j k}(s)= \begin{cases}L_{j j} s+r_{L j}+Z_{R C j}(s) & \text { if } j=k \\ L_{j k} s & \text { if } j \neq k\end{cases}
$$

and $r_{L j}$ is the series resistance of each inductance and $Z_{R C j}$ is,

$$
Z_{R C j}(s)=\left(\frac{1}{C_{o j} s}+E S R_{j}\right) \| R_{L j}
$$

$C_{o j}$ is the output capacitance, $E S R_{j}$ is the equivalent series resistor of the capacitance and $R_{L j}$ is the output load of the $j$-th output of the converter.

The function $G_{v_{e 1} v_{c}}$ corresponds to the error amplifier transfer function. This block is fully controlled by the designer and usually built with an opamp that allows to compensate and stabilise the overall control loop.

\section{OUTPUT IMPEDANCE}

To obtain the output impedance, a current perturbation, $i_{g}$ must be injected at the output. The output impedance results from measuring the output voltage perturbation 


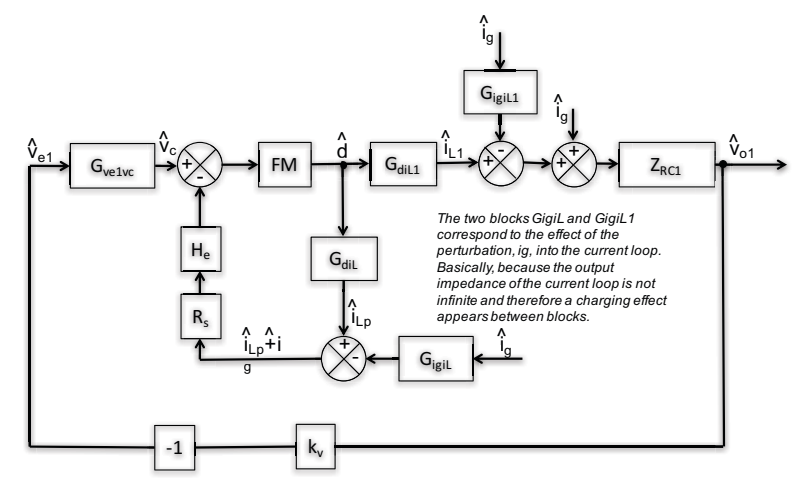

Figure 5. Small signal control block diagram of the PCM showing the current $i_{g}$ perturbation injection. This perturbation affects also the current loop with two additional blocks, $G_{i_{g} i_{L 1}}$ and $G_{i_{g} i_{L}}$ that weigh this influence.

divided by the injected current perturbation and keeping any other perturbation equal to zero.

But an additional effect must be taken into account based on the block diagram shown in Fig. 5. Although ideally the input impedance of all these blocks is infinite and their output impedance is zero, this is not true in real application. The resulting loading effect counts for additional effects of this injected perturbation at other points of the diagram, as shown in Fig. 5. A more physical explanation would be that the injected current affects the current loop, both at primary side and at secondary side. In fact the perturbation injected in output number one is coupled to all other outputs through the coupled inductor and therefore affects all other outputs. Of course, also the overall current loop is affected. This influence is described by $G_{i_{g} i_{L 1}}$ and $G_{i_{g} i_{L}}$.

Applying matrix algebra and taking into account the small signal analysis of the converter, the expression for both transfer functions can be calculated,

$$
\left.G_{i_{g} i_{L}}(s)\right|_{j=1}=Z_{R C j} \sum_{k} n_{r k}\left[\mathbf{Z}_{\mathbf{L R C}}(s)^{-1}\right]_{j k}
$$

And for the given output, the expression is,

$$
\left.G_{i_{g} i_{L j}}(s)\right|_{j=1}=\left[\mathbf{Z}_{\mathbf{L R C}}(s)^{-1}\right]_{j j} Z_{R C j}
$$

The index $j=1$ stands for injecting the signal at the first output. Both equations, Eq. 9 and Eq. 10, show that the influence of the injection into the whole system depends on the whole output impedance matrix, $\mathbf{Z}_{\mathbf{L R C}}(s)$, which, due to the inductance matrix, couples all outputs together (because it is not a diagonal matrix).

The output impedance is given by the following expression (where the dependence on $s$ of the open and closed loop transfer functions $G$ and $T$ and $H_{e}$ have been omitted in Eq. 11 for simplicity).

$$
Z_{o}(s)=Z_{R C 1} \frac{\frac{R_{s} H_{e} F M G_{d i_{L 1}} G_{i_{g} i_{L}}}{T_{i}+1}-G_{i_{g} i_{L 1}}+1}{T_{v}+1}
$$

The current loop gain $T_{i}$ is given by

$$
T_{i}(s)=R_{s} H_{e}(s) F M G_{d i_{L}}(s)
$$

and the voltage loop gain $T_{v}$ is given by

$$
T_{v}(s)=k_{v} G_{v_{e 1} v_{c}}(s) Z_{R C 1}(s) G_{v_{c} i_{L 1}}(s)
$$

where $G_{v_{c} i_{L 1}}$ is

$$
G_{v_{c} i_{L 1}}(s)=\frac{F M}{1+T_{i}(s)} G_{d i_{L 1}}(s)
$$

The magnitude of the output impedance of both, SPICE simulated circuit (using circuit shown in Fig. 8) and theoretical equations are compared in Fig. 6. It can be clearly seen that both are almost equal, what assures that the equations are right and fit the model and SPICE simulation, as both reach the same results. The expression of the output impedance when uncoupling the output inductors has also been simulated and calculated from the deduced equations, which are basically the same.

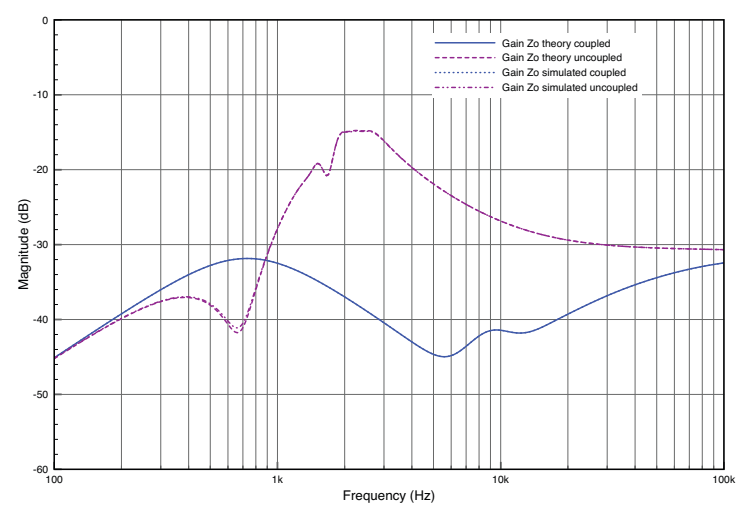

Figure 6. Magnitude of output impedance obtained from the described equations and the SPICE circuit shown in Fig. 8. Both, simulated and calculated curves are identical. The output impedance when uncoupling all outputs is also shown to demonstrate that it is worse.

It is clearly seen in Fig. 6 that the output impedance of the circuit using coupled inductance is lower than the one using uncoupled inductances. In addition, when uncoupling the inductances, the curve shows an uneven trace because 
output impedance of all different outputs add together in the primary and their different resonances are then seen by the primary peak current loop. It has to be remembered that coupling inductors together results in a single equivalent primary inductance and therefore a single resonance (see [10]), resulting in a much more even curve. A $15 \mathrm{~dB}$ improvement of the impedance comparing the peaks of both curves is reached (see Fig. 6).

\section{EXPERIMENTAL RESULTS}

Using the EM of the PCM, the output impedance has been measured. As the PCM is much more complex than the model used to study the coupling effect on multiple output converters, the results cannot be directly compared with the simulated and theoretical results shown before. An indirect procedure has been used to validate the measurements. Based on the SPICE model used in the theoretical verification, a new more detailed SPICE circuit (see Fig. 9) of the PCM has been built. This SPICE circuit includes many of the existing parasitic elements, the input filter, internal blocks of the PWM modulator, rectifying output diodes and resistance of tracks and switching devices (these switching devices are responsible of switching ON or OFF the different output voltages).

This complex SPICE circuit has been compared to the measured output impedance (see Fig. 7). It can be seen that both curves are very similar, what confirms that the base model, that demonstrates that output impedance improves when coupling output inductors together, is correct.

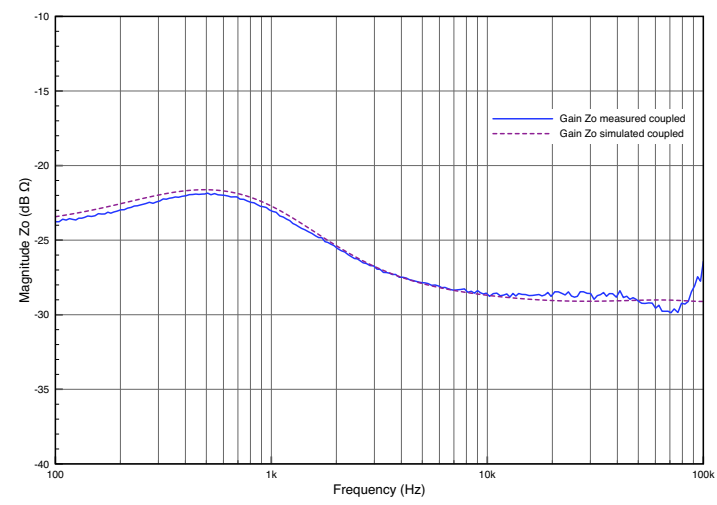

Figure 7. Output impedance measured in the real circuit and compared with simulated output impedance using a more detailed SPICE circuit (see Fig. 9) based on the already verified SPICE circuit (see Fig. 8).

The shape of the real impedance changes compared to the simulated ideal impedance shape. The main resonance peak stays at the same frequency range between, $500 \mathrm{kHz}$ and $1000 \mathrm{kHz}$. The change of this frequency is because more output capacitors have been added in the real circuit, after the ON/OFF switches. Therefore a kind of RC network (the R's come from the ESR of the capacitors and the resistances of the tracks) is placed after the output of the converter and the injection is done at this point. This is not shown in Fig. 1 but in Fig. 9. This fact increases the output impedance (about $10 \mathrm{~dB}$ which corresponds to a factor of 3.2) and also changes its shape. Therefore, the theoretical output impedance measurement is different from the real circuit, because the circuits are different. Real circuits have more parts after the output and this changes its behaviour compared to the ideal circuit.

\section{CONCLUSIONS}

This paper demonstrates that coupling output inductances in BUCK-type multiple-output converters and regulating a single output, brings as additional benefit a lower output impedance at the regulated output compared to an equivalent uncoupled converter. Other, already known benefits of coupling output inductors are mass and especially volume saving, even if the magnetic design and manufacturing of converter can be more complex.

Anyhow, it must also be stated that the improved output impedance can be slightly masked by parasitic effects found in the real circuit of the whole power converter module. The addition of the power distribution circuitry and distributed output filtering addition of output capacitors at the user side, which adds RC elements to the output path, results in a slightly different topology with a different output impedance (usually larger). As a result, the experimental measurement of the output impedance of the real system is different to the theoretical one, both in magnitude and in shape. But adding, mainly all parasitic resistances to the SPICE circuit allows to model this behaviour very well.

\section{ACKNOWLEDGMENTS}

This research has been supported with funds of the Ministerio de Economía y Competitividad of Gobierno de España and FEDER, under projects with reference:

- AYA2012-39636-C06-03

- ESP2013-47349-C6-5-R

- ESP2014-56169-C6-4-R

and partially by the Generalitat Valenciana, under project reference PROMETEO/2012/044.

\section{REFERENCES}

[1] R.W. Erickson and D. Maksimovic. A multiplewinding magnetics model having directly measurable parameters. In Power Electronics Specialists Conference, 1998. PESC 98 Record. 29th Annual IEEE, volume 2, pages 1472-1478, 1998. 


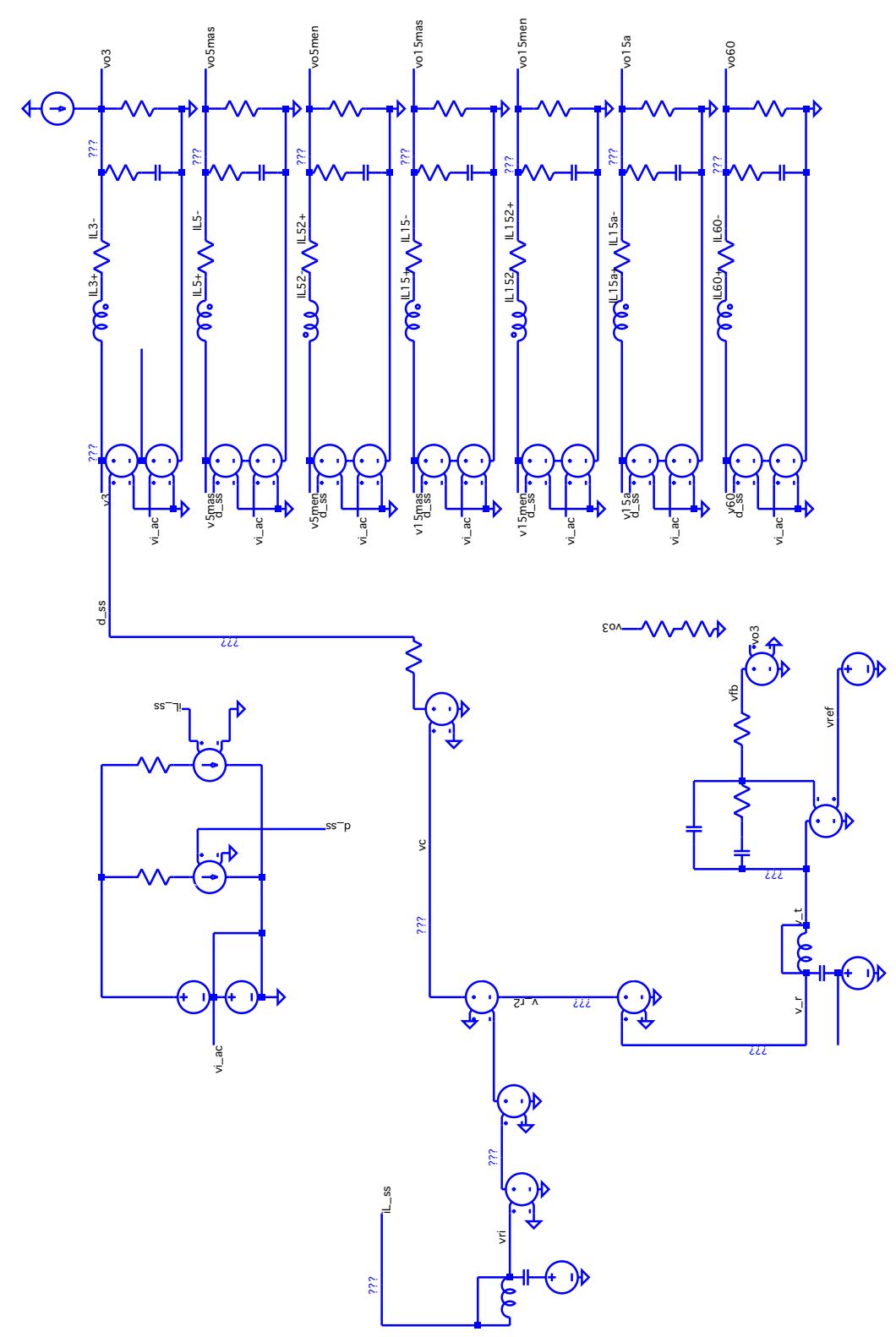

Figure 8. "Ideal" SPICE circuit used to simulate the ideal behaviour of the PCM. The frequency response is shown in Fig. 6.

[2] H. B Shin, J. G Park, S-K Chung, H. W Lee, and T.A. Lipo. Generalised steady-state analysis of multiphase interleaved boost converter with coupled inductors. Electric Power Applications, IEE Proceedings -, 152(3):584-594, 2005.

[3] H. B Shin, E.-S. Jang, J. G Park, H. W Lee, and T.A. Lipo. Small-signal analysis of multiphase interleaved boost converter with coupled inductors. Electric Power Applications, IEE Proceedings -, 152(5):1161-1170, 2005.

[4] V. Vorperian. The effect of the magnetizing inductance on the small-signal dynamics of the isolated cuk converter. Aerospace and Electronic Systems, IEEE Transactions on, 32(3):967-983, July 1996.
[5] D. Maksimovic, R.W. Erickson, and C. Griesbach. Modeling of cross-regulation in converters containing coupled inductors. Power Electronics, IEEE Transactions on, 15(4):607-615, 2000.

[6] Qing Chen, F.C. Lee, and M.M. Jovanovic. Smallsignal analysis and design of weighted voltage control for a multiple-output forward converter. Power Electronics, IEEE Transactions on, 10(5):589-596, 1995.

[7] Qing Chen, F.C. Lee, and M.M. Jovanovic. Current-mode control for multiple-output converters. Aerospace and Electronic Systems, IEEE Transactions on, 32(4):1412-1420, 1996.

[8] M. Veerachary. Power tracking for nonlinear 


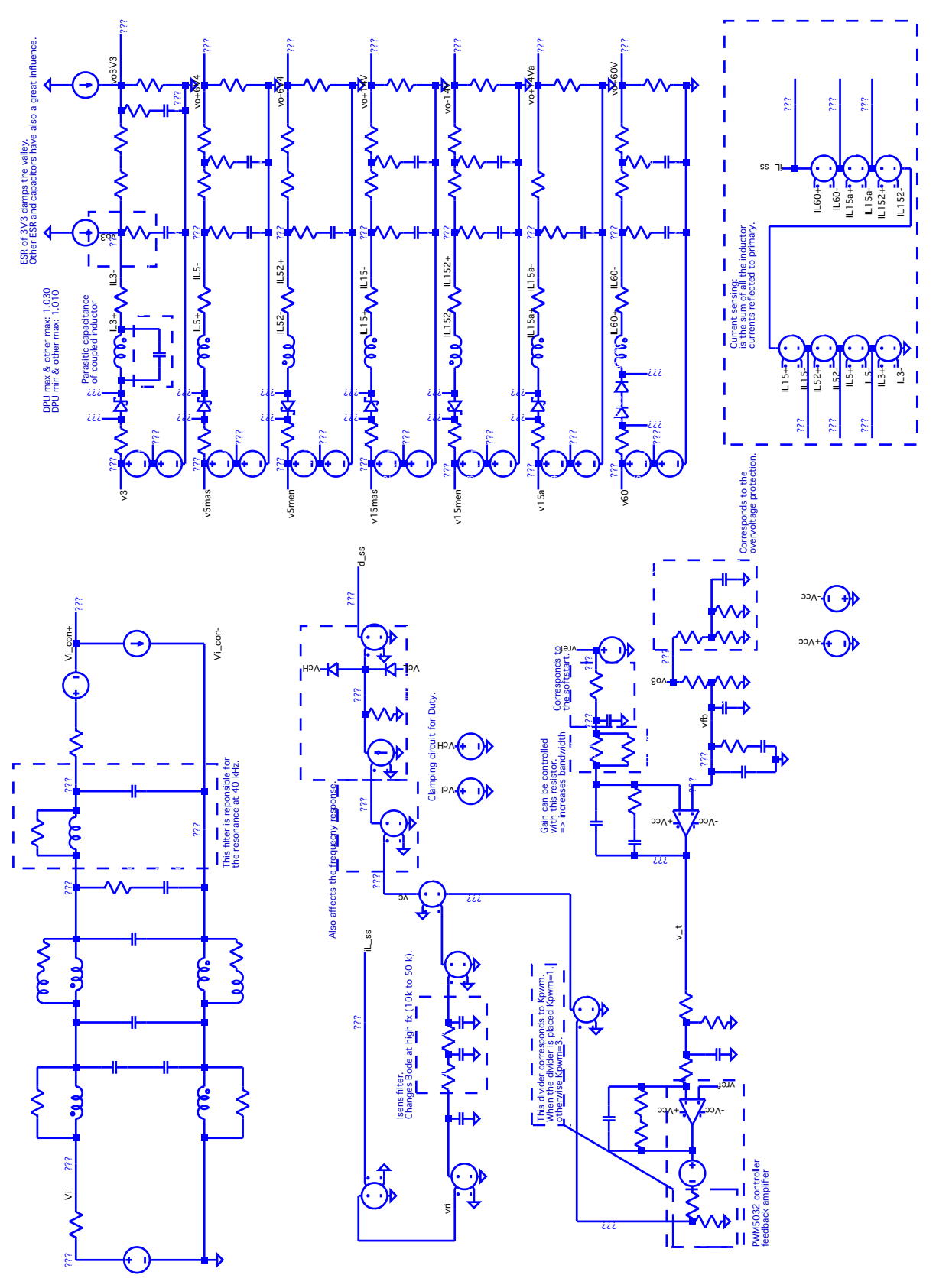

Figure 9. "Real" SPICE circuit used to simulate the real behaviour of the PCM. The frequency response is shown in Fig. 7 and compared to the measured curve. The values of the different parameters are not shown in this picture.

pv sources with coupled inductor sepic converter. Aerospace and Electronic Systems, IEEE Transactions on, 41(3):1019-1029, July 2005.

[9] P. Alou, J.A. Cobos, O. Garcia, R. Prieto, and J. Uceda. Coupling inductors for supplying pulsating loads with $\mathrm{dc} / \mathrm{dc}$ converters. In Power Electronics Specialists Conference, 1998. PESC 98 Record. 29th Annual IEEE, volume 2, pages 1790-1795 vol.2, May 1998.

[10] E. Sanchis-Kilders, A. Ferreres, E. Maset, J.B. Ejea, J.L. Gasent-Blesa, V. Esteve, J. Jordan, A. Garrigos, and J.M. Blanes. On the design of a multiple-output $\mathrm{dc} / \mathrm{dc}$ converter for the phi experiment on-board of solar orbiter. In Applied Power Electronics Conference and Exposition (APEC), 2013 Twenty-Eighth Annual IEEE, pages 3305-3310, March 2013.

[11] B. Hesterman. Analysis and modelling of magnetic coupling. Technical report, Advanced Energy Industries, www.denverpels.org, 2007.

[12] Yilmaz Tokad and Myril B. Reed. Criteria and tests for readability of the inductance matrix. American Institute of Electrical Engineers, Part I: Communication and Electronics, Transactions of the, 78(6):924-926, 1960. 